This item was submitted to Loughborough's Research Repository by the author.

Items in Figshare are protected by copyright, with all rights reserved, unless otherwise indicated.

\title{
A tensor-based catheter and wire detection and tracking framework and its clinical applications
}

\section{PLEASE CITE THE PUBLISHED VERSION}

https://doi.org/10.1109/tbme.2021.3102670

\section{PUBLISHER}

Institute of Electrical and Electronics Engineers (IEEE)

VERSION

AM (Accepted Manuscript)

\section{PUBLISHER STATEMENT}

Personal use of this material is permitted. Permission from IEEE must be obtained for all other uses, in any current or future media, including reprinting/republishing this material for advertising or promotional purposes, creating new collective works, for resale or redistribution to servers or lists, or reuse of any copyrighted component of this work in other works.

\section{LICENCE}

\section{All Rights Reserved}

\section{REPOSITORY RECORD}

Ma, Yingliang, Diwei Zhou, Lei Ye, Richard James Housden, Ansab Fazili, and Kawal Rhode. 2021. "A Tensor-based Catheter and Wire Detection and Tracking Framework and Its Clinical Applications". Loughborough University. https://hdl.handle.net/2134/15129102.v1. 


\title{
A Tensor-based Catheter and Wire Detection and Tracking Framework and Its Clinical Applications
}

\author{
YingLiang Ma, Diwei Zhou, Lei Ye, R. James Housden, Ansab Fazili and Kawal S. Rhode
}

\begin{abstract}
Objective: Catheters and wires are used extensively in cardiac catheterization procedures. Detecting their positions in fluoroscopic X-ray images is important for several clinical applications such as motion compensation and co-registration between 2D and 3D imaging modalities. Detecting the complete length of a catheter or wire object as well as electrode positions on the catheter or wire is a challenging task. Method: In this paper, an automatic detection framework for catheters and wires is developed. It is based on path reconstruction from image tensors, which are eigen direction vectors generated from a multiscale vessel enhancement filter. A catheter or a wire object is detected as the smooth path along those eigen direction vectors. Furthermore, a real-time tracking method based on a template generated from the detection method was developed. Results: The proposed framework was tested on a total of 7,754 X-ray images. Detection errors for catheters and guidewires are $0.56 \pm 0.28 \mathrm{~mm}$ and $0.68 \pm 0.33 \mathrm{~mm}$, respectively. The proposed framework was also tested and validated in two clinical applications. For motion compensation using catheter tracking, the 2D target registration errors (TRE) of $1.8 \mathrm{~mm} \pm 0.9 \mathrm{~mm}$ was achieved. For coregistration between 2D X-ray images and 3D models from MRI images, a TRE of $2.3 \pm 0.9 \mathrm{~mm}$ was achieved. Conclusion: A novel and fully automatic detection framework and its clinical applications are developed. Significance: The proposed framework can be applied to improve the accuracy of imageguidance systems for cardiac catheterization procedures.
\end{abstract}

Index Terms - Cardiac catheterization procedures, Motion correction, Wire detection, Catheter detection, Image-guided intervention, Electrophysiology

\section{INTRODUCTION}

$\mathrm{M}$ inimally-invasive cardiac interventional procedures such as the treatment of heart failure, structural heart disease and cardiac electrophysiology (EP) procedures are usually guided using X-ray fluoroscopy. The procedures generally involve catheters and wires, which are made from high density materials, and so are visible in the X-ray images. However, soft tissues have poor contrast under X-ray. To aid

Manuscript received Feb. xx, 2021. This work was supported by the National Institute for Health Research (NIHR) Biomedical Research Centre based at Guy's and St Thomas' NHS Foundation Trust and King's College London and a NIHR research grant II-LB-0814-20001.

Dr. YingLiang $\mathrm{Ma}$ is with School of Computing, Electronics and Mathematics, Coventry University, CV1 5FB, United Kingdom (corresponding author: e-mail: yingliang.ma@ coventry.ac.uk). navigation and overcome the lack of soft tissue contrast, threedimensional (3D) roadmaps can be overlaid onto X-ray images to add anatomical information. 3D roadmaps can be generated from pre-procedural computed tomography (CT) images [1]-[3] and magnetic resonance (MR) images [4] [5]. Modern cardiac interventional X-ray systems can also perform rotational X-ray angiography, which can be used to form 3D roadmaps for guiding procedures [6][7]. Detecting the position of catheters or wires in X-ray images is important for several clinical applications. For example, a major limitation of 3D roadmapping technologies is that overlaid models remain static and do not move with the patient's respiratory and cardiac motions. Using the position of a catheter or a wire object which is inserted into the narrow blood vessels (e.g. coronary sinus) of the heart will help compensate the motions of the heart as the catheter or wire moves together with the heart. Catheter or wirebased motion compensation can largely reduce the twodimensional (2D) registration error as, in some cases, respiratory motion can cause a two-dimensional (2D) registration error of over $14 \mathrm{~mm}$ [8]. For co-registration between a 2D X-ray image and 3D roadmap, an automatic registration method could be developed if the position of a catheter or a wire object inside the narrow blood vessel is detected. The automatic method will use the centerline of the detected object to align with the centerline of the narrow blood vessel models in the roadmaps. Moreover, localizing wires and catheters can facilitate optimal collimation [9]. Collimators control the field of view by using thick blades to block part of the X-ray radiation leaving the source to the patient. Detecting the locations of wires or catheters can automatically narrow down the region of interest.

For wire and catheter detection and tracking, the majority of work is only focused on tracking which requires manual initialization or pre-defined models. Pre-defined geometric models were used in [10][11] to track the lasso catheter and a deformed B-spline model was implemented to track guidewires in X-ray image [12]. Recently, several machine learning-based

Dr. Diwei Zhou and Lei Ye are with Department of Mathematical Sciences, School of Science, Loughborough University, Loughborough, LE11 3TU, United Kingdom.

Ansab Fazili is with Department of Cardiology, Lister Hospital, Stevenage, United Kingdom.

Dr. R. James Housden and Prof. Kawal Rhode are with School of Biomedical Engineering and Imaging Sciences, King's College London, St. Thomas' Hospital, London, SE1 7EH, United Kingdom. 
detection and tracking methods were developed. Yatziv et al. [13] presented a catheter detection method based on cascade classifiers. Although their method achieved 3.97\% detection failure rate, it only detected the catheter tip electrode and could fail when a thicker lasso catheter was nearby. Zhou et al. [14] utilized a fast attention recurrent network to detect the endpoint of the guidewire tip, and Ambrosini et al. [15] used a convolutional neural network to detect guide catheters in liver catheterization procedures. Wu et al. [16] proposed a learningbased approach to temporally track and detect catheter electrodes in fluoroscopy sequences, but it only tracks electrodes and requires manual initialization. Very few detection methods have been published to fully automatically localize the positions of both catheters and wire objects as well as electrode positions on the catheter or wire. Ma et al. [17] [18] have developed a detection method based on blob detection and a vessel filter. However, these methods only work on catheters with electrodes or very rigid wires. The success rate of these methods will be reduced when the target object is overlapping with other wires. Navab et al. [19] applied a machine learning approach on randomly generated deformable models to extract wire objects. However, this was not tested on cases where wires have a sudden and large deformation movement.

All these existing methods do not meet all requirements of our clinical applications. In addition, we are not aiming to design and implement a computational framework to compete with existing methods. Instead, we are focusing on the requirements of our applications to deliver the necessary performance and accuracy. The requirements are (1) no manual initialization, (2) real-time tracking speed, (3) landmark (electrode) tracking on the catheter and (4) tracking the fulllength of catheters or wire objects. This is to facilitate automatic co-registration between $2 \mathrm{D}$ and $3 \mathrm{D}$ imaging modalities so that there are enough geometric data available for registration. If only electrodes on the catheter were tracked, the positions of electrodes can only generate part of geometric data of the target catheter (from the catheter-tip electrode to the proximate electrode). Therefore, tracking the whole length of the target catheter will provide more geometric data.

In this paper, we propose a novel catheter and wire detection and tracking framework, which is fully automatic and can be used in several clinical applications. It is clinically robust and has minimal interference with the routine clinical workflow. For detection, a multiscale vessel enhancement filter is applied to the X-ray image, which generates eigen direction vectors in the areas of the wire-like objects. The catheter or wire object is detected as a smooth path constructed by using a tensor path reconstruction algorithm. Image tensors at each pixel in the image can be expressed by eigenvalues $\left(\lambda_{1}, \lambda_{2}\right)$ and eigenvectors $\left(v_{1}, v_{2}\right)$. An image tensor is considered as isotropic when its eigenvalues are nearly equal $\left(\lambda_{1} \approx \lambda_{2}\right)$ and as anisotropic when they are significantly different in magnitude $\left(\lambda_{1}>\lambda_{2}\right)$. For an anisotropic pixel, its $v_{l}$ is recognized as the principal eigenvector, which is used to determine the possible wire direction at that point. A complete catheter or wire object therefore can be reconstructed by analyzing the tensor field in the image. To improve the success rate, the similarity scores are calculated among detected objects in the first 5 frames of the video stream. The detected object with the highest score is selected as the tracking template. As for both motion compensation and co-registration between 2D and 3D imaging modalities, a static catheter or wire object is required so that it remains stationary except for respiratory and cardiac motions. Therefore, the tracking method only has to find the reconstructed path with a similar shape to the tracking template.

For the application of motion compensation, the coronary sinus (CS) catheter is used, as the CS catheter is widely used in cardiac catheterization procedures and it remains stationary most of the time during the procedure. According to [8], the majority of the motion of the heart caused by respiratory motion is translational, and the majority of the translational motion of the heart is in the superior-inferior direction (head to foot direction). Although a 2D translational motion model is less accurate than more comprehensive motion models such as patient specific affine motion models, it does not require additional patient specific data and lengthy computation, and it can be used in any cardiac procedure. Therefore, we developed 2D landmark tracking on a CS catheter to correct the respiratory motion. The landmark is an electrode which is clearly visible in $\mathrm{X}$-ray images and it can be reliably tracked even at very low radiation doses [20]. For the application of automatic coregistration between $2 \mathrm{D}$ and $3 \mathrm{D}$ imaging modalities, a wire or a catheter in narrow blood vessel is used. For example, a CS catheter is used as the registration object in cardiac electrophysiological (EP) procedures, where it is inserted into the narrow coronary sinus.

The main contribution of this manuscript is the novel catheter and wire tracking framework and its clinical applications. The framework was tested on a large clinical data set and validated in two clinical applications.

\section{IMAGE ACQUISITION AND IMAGE OVERLAY}

Image-guided cardiac catheterization procedures were carried out using a mono-plane flat-panel cardiac X-ray system at St. Thomas' Hospital, London. A total of 108 image sequences (8,364 images) were acquired in 39 different clinical cases. 19 patients (a total of 3521 images were acquired) underwent ablation procedures for the treatment of atrial fibrillation (AF), and for each patient anatomical imaging was performed prior to the procedure. 13 of these patients underwent cardiac MRI (Philips Achieva) and 6 patients underwent CT imaging (Philips Brilliance). 15 of the other cases (2117 images) were from cardiac resynchronization therapy (CRT) procedures. The remaining 5 cases (2726 images) were from cardiac catheterization procedures for treating congenital heart defects (CHD).

\section{A. Imaging protocol}

For patients undergoing MRI, a high resolution freebreathing whole heart MRI scan was acquired (3D balanced TFE, respiratory gated at end-expiration, cardiac triggered and gated at late diastole).

For patients undergoing CT, a retrospectively-gated CT acquisition was performed under end-inspiratory breath-hold 
and the $80 \%$ R-R interval reconstruction was used for subsequent processing.

The image segmentation was performed using a shape model-based automatic segmentation algorithm for CT [21] and for MR [22]. The 3D anatomical models can be derived from segmented 3D image data and will be used in fluoroscopy overlay.

\section{B. Fluoroscopy overlay}

The interventional guidance platform was a prototype offline 2D-3D overlay software. The software allowed the registration of pre-procedural MR/CT-derived anatomical models onto an $\mathrm{X}$-ray fluoroscopy sequence. This was done manually through multiple-view X-ray images. As the guidance platform also received the synchronized positions of the $\mathrm{C}$-arm and $\mathrm{X}$-ray table with the $\mathrm{X}$-ray fluoroscopy sequence, alignment between pre-procedural anatomical models and X-ray images was then maintained irrespective of changes in the system geometry.

\section{MethodS}

\section{A. Tensor-based catheter and wire detection method}

The proposed detection method is divided into three steps. 1) Creating a tensor field using a multiscale vessel enhancement filter. 2) Performing fibre detection according to the tensor field. 3) Reconstructing the wire path by analyzing fibres found. Figure 1 gives the overall workflow for detecting catheters and wires.

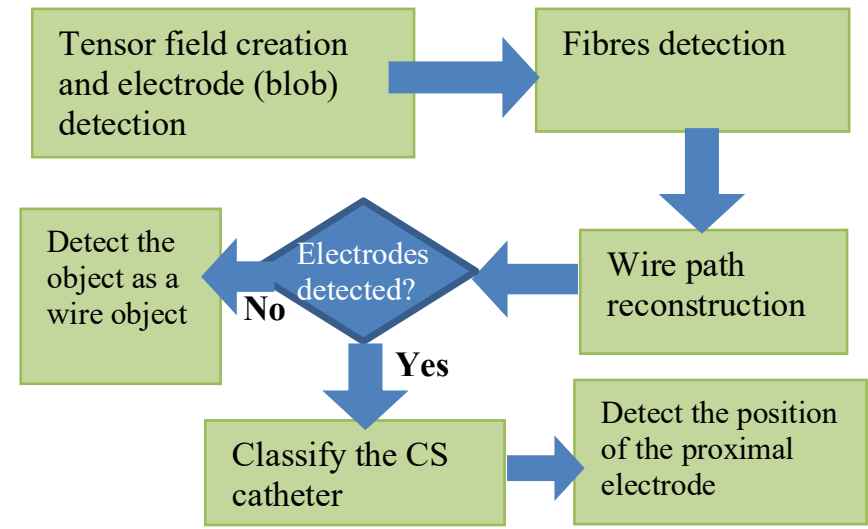

Fig. 1. Catheter and wire detection workflow

\section{1) Creating a tensor field}

In order to create a tensor field in the X-ray images, a multiscale vessel enhancement filter [23] is used to calculate the eigenvalues and eigenvectors on wire-like structures in the image. This involves three steps. Firstly, the image is smoothed by applying a Gaussian kernel of the appropriate scale $s$. The smoothed image $L(X, s)$ is computed as $L(X, s)=L(X) *$ $G(X, s)$, where * is the convolution operator, $G(X, s)$ is the Gaussian kernel and $L(X)$ is the input image. Step 2 is to form and decompose the $2 \times 2$ Hessian matrix at every image pixel. The Hessian matrix $H_{X, S}$ is defined as

$$
H_{X, s}=\left[\begin{array}{ll}
L_{x x}(X, s) & L_{x y}(X, s) \\
L_{y x}(X, s) & L_{y y}(X, s)
\end{array}\right]
$$

where $L_{x y}(X, s)=\frac{\partial}{\partial x}\left(\frac{\partial}{\partial y} L(X, s)\right)$, and the other terms are defined similarly. The final step is to extract both eigenvalues and eigenvectors by decomposing the Hessian matrix $H_{X, S}$. In order to reduce computational complexity for the following path reconstruction and achieve real-time detection speed, the image is binarized using Otsu's method [24], after applying the vessel enhancement filter. Otsu's method is a non-parametrized and adaptive algorithm as it automatically determines the thresholding level based on minimizing the intra-class variance. Otsu's method has previously been used together with a vessel enhancement filter for coronary sinus segmentation on X-ray images [25]. In this way, eigenvalues are set to zero for the areas outside the binarized wire-like structures and they will be excluded from the path reconstruction algorithm. Otsu thresholding greatly reduces the search-space of the wire-path reconstruction algorithm. For example, in figure 2, the remaining tensors is only $7.2 \%$ of all tensors after applying Otsu thresholding. The wire path reconstruction algorithm can be repeated several times in order to extract multiple wire-like objects and ignore the short ones like the ultrasound probe and bone structures. Further to reduce the computational load, eigenvalues and eigenvectors are only sampled every 4 pixels in each direction, so that only a small percentage (less than $6.25 \%$ ) of all eigenvalues and eigenvectors will participate in the path reconstruction algorithm. Figure 2 gives an example of creating the tensor field.

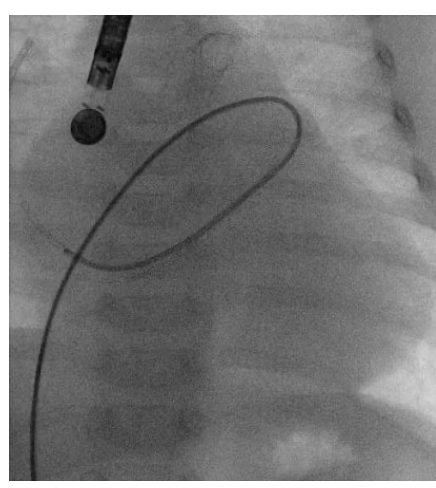

(a)

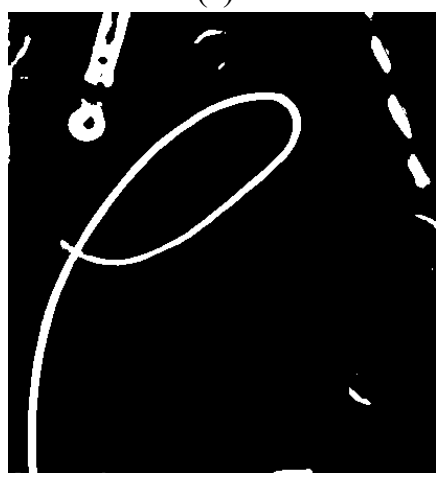

(c)

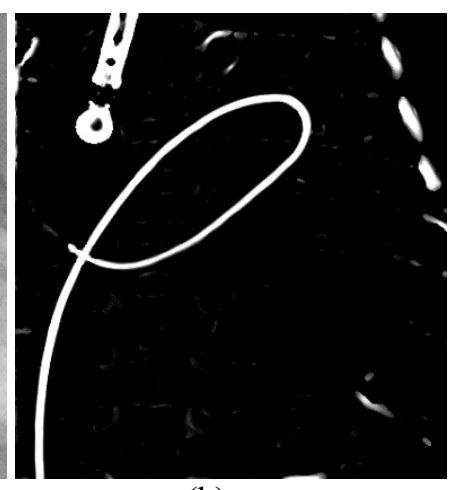

(b)

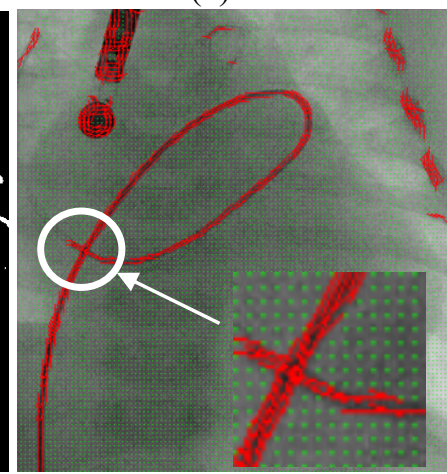

(d)
Fig. 2. (a) The original X-ray image. (b) Image after applying the vessel enhancement filter. (c) Image after binarization using Otsu's method. (d) The tensor field. Green dots are the eigenvectors excluded from participating in the path reconstruction algorithm. Red lines are the directions of the eigenvectors.

The vessel enhancement filter not only can increase the 
visibility of wire-like structures in the image but also can detect the position of catheter electrodes. As $H_{X, S}$ is a $2 \times 2$ matrix, there are two eigenvalues $\left(\lambda_{1}, \lambda_{2}\right)$ and two eigenvectors at every image pixel. To classify any local structures in the image, the ratio between two eigenvalues $R=\lambda_{1} / \lambda_{2}$ is calculated. If $R \approx$ 1 , detected structures will be blob-like structures (electrodes). Otherwise, they will be wire-like structures.

The image tensor $D$ at each pixel is defined by

$$
D=\left[\begin{array}{ll}
v_{1} & v_{2}
\end{array}\right]\left[\begin{array}{cc}
\lambda_{1} & 0 \\
0 & \lambda_{2}
\end{array}\right]\left[\begin{array}{l}
v_{1} \\
v_{2}
\end{array}\right]
$$

where $\lambda_{1} \geq \lambda_{2} \geq 0$. The two unit column vectors $v_{1}$ and $v_{2}$ are the corresponding orthogonal eigenvectors. The eigenvector $v_{1}$ is called the principal eigenvector which is associated with the larger eigenvalue $\lambda_{1}$. By definition, the image tensor $D$ is a 2 by 2 symmetric positive semi-definite real matrix. The dissimilarity between two image tensors $D\left(X_{i}\right)$ and $D\left(X_{j}\right)$ can be quantitatively described using a distance function. For example, the Euclidean distance between two image tensors is given by

$d_{E}\left(D\left(X_{i}\right), D\left(X_{j}\right)\right)=\left\|D\left(X_{i}\right)-D\left(X_{j}\right)\right\|$

where $\|A\|=\sqrt{\operatorname{trace}\left(A^{T} A\right)}$ is the Euclidean norm (also known as the Frobenius norm). It is more natural to choose nonEuclidean distance functions (such as the root Euclidean distances) due to the positive semi-definiteness of tensor data [26][27].

An anisotropy measure is a useful tool to capture the directionality of $D$. An image tensor is anisotropic if $\lambda_{1} \gg$ $\lambda_{2}$ and it is isotropic if $\lambda_{1}=\lambda_{2}$. For example, an Anisotropy Index (AI) of $D$ can be defined by

$A I(D)=\frac{\sqrt{2\left[\left(\lambda_{1}-\bar{\lambda}\right)^{2}+\left(\lambda_{2}-\bar{\lambda}\right)^{2}\right]}}{\sqrt{\lambda_{1}{ }^{2}+\lambda_{2}{ }^{2}}}$

where $\bar{\lambda}=\left(\lambda_{1}+\lambda_{2}\right) / 2 . A I(D)$ ranges from 0 for fully isotropic to 1 for fully anisotropic. To reduce the computational cost of equation (4), as it will be calculated for every tensor in the image, it can be simplified to

$A I(D)=\frac{\lambda_{1}-\lambda_{2}}{\sqrt{\lambda_{1}{ }^{2}+\lambda_{2}{ }^{2}}}$

\section{2) Fibre detection}

Given a tensor field $D\left(X_{1}\right), D\left(X_{2}\right), \ldots, D\left(X_{N}\right)$ where $N$ is the total number of pixels in an image, the algorithm to track wire sections is illustrated below

1. Start from a seed pixel $X_{0}$ with AI value greater than threshold $T_{l}$ and search for a wire along both sides of its principal eigenvector $v_{1}\left(X_{0}\right)$;

2. Take $v_{1}$ at $X_{0}$ as the current wire search direction and pixel;

3. Calculate the angles $\theta$ between $v_{1}\left(X_{0}\right)$ and the offset vectors $v_{\text {offset }}$, which are the centre connections from $v_{1}$ to each adjacent pixel respectively, then sort the results in ascending order;

4. Find the adjacent pixels whose $\theta$ is less than the tolerance threshold $T_{2}$;
5. Compare the tensor similarity between $X_{0}$ and the adjacent pixels found in step 4 by calculating root Euclidean distance;

6. Find the most similar neighbour pixel $X_{n}$ to the current pixel along the wire. If no satisfactory pixel is found, go to step 11;

7. Check the AI at $X_{n}$. If it is less than the threshold $T_{l}$, go to step 11;

8. Calculate the inner product of $v_{1}\left(X_{0}\right)$ and the principal eigenvector $v_{1}\left(X_{n}\right)$ at $X_{n}$. Swap the sign of $v_{1}\left(X_{n}\right)$ if the result is negative;

9. Check the turning angle between $v_{1}\left(X_{0}\right)$ and $v_{1}\left(X_{n}\right)$, if it is greater than threshold $T_{3}$, go to step 11;

10. Assign $X_{n}$ to $X_{0}$, assign $v_{1}\left(X_{n}\right)$ to $v_{1}\left(X_{0}\right)$, and go to step 2;

11. Restart the search from the original pixel $X_{0}$, swap the sign of $v_{1}\left(X_{0}\right)$ at $X_{0}$, and perform detection in the reverse direction from step 2 again. If detection has been completed for both directions of the seed pixel, go to step 12;

12. The fibre detection is completed by combining the detection results of both directions from the seed point together.

13. If all seed pixels have been processed, stop, otherwise go to step 1 .

Here, $T_{1}=0.0001 . T_{2}=40$ and it is the tolerance angle for fibre tracking. $T_{3}=45$ and it is the turning angle for fibre tracking. $T_{1}, T_{2}$ and $T_{3}$ are optimized in the training dataset and those values are fixed for testing all image sequences. For step (7), when a catheter is crossing itself, the wire tracking algorithm could either continue or stop, which depends on the actual tensor data. In the scenario of stopping, the wire can be reconnected to its remaining part according to the algorithm of "Reconstructing whole wire path".

\section{3) Reconstructing whole wire path}

Based on the fibres found in the image, the complete path of a catheter or a wire can be reconstructed. The wire path reconstruction algorithm connects fibres found in Step 2 to construct a long wire. This can be repeated several times in order to extract multiple wire objects.

The algorithm is listed as follows:

1. Sort all the fibres according to length in descending order;

2. Start from the longest fibre, assume it is part of the final wire $W$, and add its ID into merge list MList;

3. Find the next fibre $f$ if it is not in MList and then calculate the distances and link directions between the 2 endpoints of $W$ and 2 endpoints of $f$, respectively;

4. If the distance is less than Range threshold $R_{l}$, the angle between $W$ and $f$ is less than Direction threshold $R_{2}$, and the link angle between $W$ and $f$ is less than Link threshold $R_{3}$, then merge $f$ into $W$. Add $f$ ID into MList;

5. Repeat step 3 until all fibres have been processed;

6. Smooth $W$ by removing obtuse angle connections. 
Our method can not handle the sharp link angle (angle $<90$ ) between $\mathrm{W}$ and $\mathrm{f}$. In some angles of X-ray system, a smooth guidewire could be projected as a sharp-turning wire object. In this case, the path reconstruction algorithm will stop at the location of the sharp turning. $R_{1}=25$ and it is the distance range for connecting broken fibres. $R_{2}=30$ and it is the turning angle for connecting broken fibres. $R_{3}=30$ and it is the angle between the linking line and 2 broken fibres. $\mathrm{R}_{1}, \mathrm{R}_{2}$ and $\mathrm{R}_{3}$ are optimized in the training dataset and those values are fixed for testing all image sequences. The threshold $R_{2}$ is for verifying the turning angle between the two wires $W$ and $f$. Reconnection will be abandoned if the turning angle is greater than $R_{2}$.

\section{B. The tracking methods for clinical applications}

\section{1) Motion compensation application}

For the motion compensation application, electrode catheters are the ideal target objects as they have several electrodes which are highly visible in X-ray images. The position of an electrode can be used to drive $3 \mathrm{D}$ roadmaps to follow the respiratory and cardiac motions in X-ray images. To track the positions of electrodes, we used a by-product of the vessel enhancement filter: electrode (blob) positions can be detected as image pixels where the ratio between two eigenvalues is close to $1.0\left(\lambda_{1} \approx\right.$ $\lambda_{2}$ ). The vessel enhancement filter is used for creating a tensor field (described in section III.A(1)) and enabling blob detection adds no additional computational cost for the filter as the eigenvalues are always computed inside the filter. An example of blob detection can be found in figure 3 .

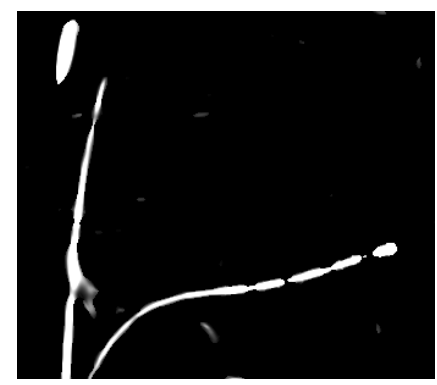

(a)

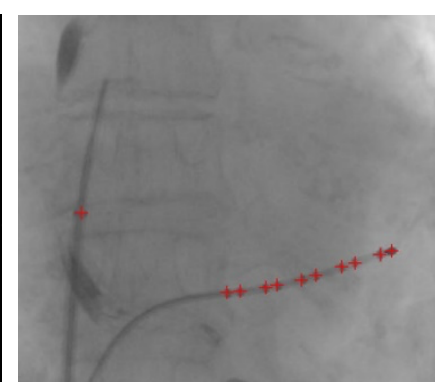

(b)
Fig. 3. Blob detection result. (a) Image after applying the vessel enhancement filter. (b) Blob positions (red crosses). The threshold for $\lambda_{1} / \lambda_{2}$ is 0.8 and $D_{\text {gap }}$ is 13 pixels.

In cardiac catheterization procedures, coronary sinus (CS) catheters are often used as diagnostic catheters and the most commonly used CS catheters are the 10-electrode or 4-electrode catheter. The presence of electrodes was the unique feature that was used to separate the CS catheter from other catheters, instruments and ECG leads. A combination of wire detection and the electrode pattern provides a robust way to detect the CS catheter inside X-ray images. The detection workflow for 10electrode CS catheter is as follows:

(1) Run the tensor-based wire detection method. In the step of tensor field creation, blob detection is performed using the vessel enhancement filter (no addition computational cost).

(2) Cluster blobs on the detected wire based on the maximum distance $D_{\max }$. $D_{\max }$ is defined as twice the length of the distance $D_{\text {gap }}$ between two neighbouring electrodes. $D_{\text {gap }}$ was obtained by physical measurement of a CS catheter and it is 5 $\mathrm{mm}$ or 13 pixels based on a pixel to $\mathrm{mm}$ ratio of 0.385 .

(3) Detect the CS catheter as the wire with a cluster of 9 or more blobs (electrodes). The reason for using 9 instead of 10 blobs as the threshold is to accommodate some cases of missed blob detection in the tip of the catheter where two electrodes are close to each other.

Our method can work with the 4-electrode CS catheter or other types of catheters as long as the catheter has electrodes. Finally, the $2 \mathrm{D}$ position of the proximal electrode of the CS catheter was used for the motion compensation application. The reason for choosing the proximal electrode is that it has less cardiac cycle motion and has lower detection errors than the catheter-tip electrode (because of occasionally overlapping with the second electrode). In order to reduce the computation cost, a tracking method is used. To generate a tracking template, the error scores are calculated among detected objects in the first 5 frames of video stream. The error score is defined as the sum of error Euclidean distances between two detected objects after the two objects are registered using iterative closest point (ICP). The detected object in one frame and other detected objects in other 4 frames were registered using ICP and the sum of all error scores were calculated. Repeat the process for all 5 frames. The one with the lowest score (sum) will be chosen as the tracking template. After the template is obtained, in the subsequent frames, a simplified version of the wire reconstruction algorithm is used to find a target object with a similar shape to the tracking template. The simplified algorithm starts with the selecting one of the detected fibres which is closed to the image boundaries and also close to the tracking template. As all catheters are inserted from blood vessels in the arms, legs or neck into the heart and the catheter inserting point is outside the field of view of X-ray images when the procedure focuses on the heart area, the end of the catheter will appear on one of image edges on X-ray images. The rest of the catheter can then be reconstructed by selecting detected fibres with the minimum angle difference and the minimum distance to the tracking template. Figure 4 illustrates an example. The angle difference is defined as the angle between the tangent vector from the template and the tangent vector from the tracked object.

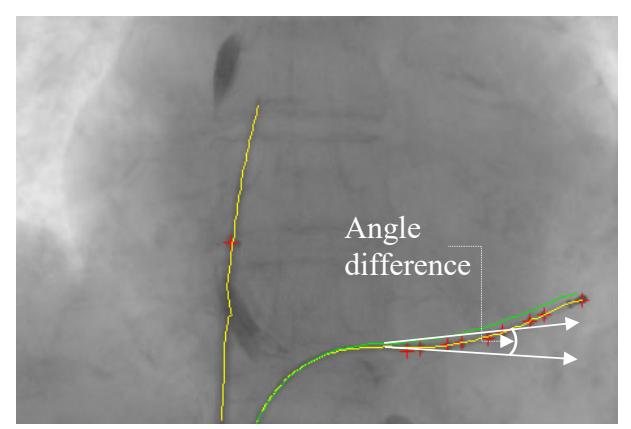

Fig. 4. Wire reconstruction using the tracking template. Red crosses are the detected blob positions. Yellow lines are the detected wire or catheter. The green line is the tracking template.

\section{2) $2 D$-3D registration application}

For the registration application, a catheter or guidewire was used to align 3D models with an X-ray image automatically. The method was implemented in a prototype overlay platform. 
During the procedure, the MR/CT-derived 3D model was imported and overlaid on the live X-ray image to guide the cardiologist. To register the 3D model with the 2D X-ray image, the transformation matrix ( $T_{\text {model_to_Xray }}$ ) needs to be calculated. It consists of a rigid body transformation matrix $T_{\text {rigid }}$ and a projection matrix $T_{\text {proj }}$.

$T_{\text {model_to_Xray }}=T_{\text {proj }} T_{\text {rigid }}$

$T_{\text {proj }}$ transforms from 3D X-ray C-arm space to 2D X-ray image space. This can be calculated by using the intrinsic parameters of the X-ray system [28]. $T_{\text {rigid }}$ transforms from $3 \mathrm{D}$ model space (MR or CT image space) to 3D X-ray C-arm space. It can be defined as

$$
T_{\text {rigid }}=T_{\text {model_to_bed }} T_{\text {bed_to_Carm }}
$$

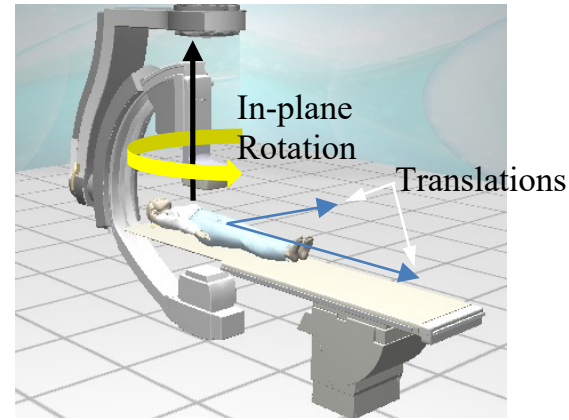

Fig. 5. Illustration of translations and in-plane rotation on the X-ray bed.

$T_{\text {bed_to_Carm }}$ transforms from 3D X-ray bed space to 3D X-ray $\mathrm{C}$-arm space. It can be calculated using positions and orientations of the X-ray bed and C-arm, which are tracked in real-time in our system. $T_{\text {model_to_bed }}$ transforms from 3D model space to 3D X-ray bed space. If the X-ray bed is aligned with the bed in the MR or CT scanner, $T_{\text {model to bed }}$ can be calculated using parameters in the DICOM header of the MR or CT images. In reality, because the way that the patient lies in the MR/CT bed may be different to the X-ray bed, a correction transformation should be done in the registration pipeline. The majority of corrections transformations are small translations and in-plane rotation on the bed. Figure 5 gives an illustration. In our X-ray system, $T_{\text {proj }}$ is generated by the system software. To maintain the accuracy of $T_{p r o j}$, the X-ray system are calibrated every few years by a service engineer and the accuracy is well below $1 \mathrm{~mm}$. $T_{\text {bed to_carm }}$ is also provided by the system software in real-time and they are generated by using position sensors in C-arm and patient bed. Again, the accuracy is well below $1 \mathrm{~mm}$. The accuracy of $T_{\text {model_to bed }}$ depends on how patient lies on X-ray bed and $T_{\text {model_to_bed }}$ can be corrected using our automatic 2D-3D registration method.

To automatically calculate the small translations and in-plane rotation, a catheter or guidewire was used to match with the centerline of the narrow blood vessel. First, a 3D thinning method for binary images is used to extract the center pixels of a blood vessel [29] from the result of segmentation of the targeted heart vessels. The target heart vessel is normally coronary sinus (CS) as it is a narrow blood vessel. CS is segmented manually by a clinician. Then a spline curve is fitted to the center pixels to create a smooth centerline. Finally, the detected catheter or guidewire which is located inside the targeted heart vessel is registered with the smoothed centerline of the projected targeted heart vessel using ICP. The electrode catheter could be recognized automatically if there are more than one catheters presented in the image. For examples, the CS catheter which is normally inserted into CS can be recognized by the pattern of electrodes. The alignment of catheter or guidewire with the centerline of the blood vessel are not limited to the CS catheter and other catheter or rigid wire can be used as long as it has sufficient length within the blood vessel.

Aligning a catheter or guidewire with the centerline of a narrow blood vessel in a single $\mathrm{X}$-ray image does not provide an accurate 2D-3D registration. Ideally, biplane images from a biplane X-ray system should be used as biplane images are acquired at the same time and they do not have respiratory or cardiac motion phase differences. However, the majority of interventional cardiology X-ray systems are mono-plane systems. Therefore, a motion gating method [30] was used to find a pair of images in similar respiratory and cardiac cycle motion phases from two short X-ray fluoroscopic image sequences, which are acquired from two different angle views using a mono-plane X-ray system. Typically, one is the posteroanterior (PA) view and the other is the left/right anterior oblique (LAO/RAO) view. We refer to this pair of images also as the biplane X-ray images. Aligning a detected catheter or guidewire with the centerline of a narrow blood vessel in the biplane X-ray images provides a 2D-3D registration.

\section{RESUlts}

108 image sequences $(8,364$ images) were acquired in 39 different clinical cases using a mono-plane X-ray system. The frame size of each sequence is $512 \times 512$, with pixel sizes between $0.368 \mathrm{~mm}$ and $0.433 \mathrm{~mm}$. The images acquired during the EP procedures contains between one catheter to four catheters and the average number of catheters is two. 610 training images from 8 image sequences were used for obtaining the optimal values for $T_{1}, T_{2}$ and $T_{3}$ as well as $R_{1}, R_{2}$ and $R_{3}$. The remaining 7754 images were used for evaluation and validation. Among the validation images, 3221 images are from $\mathrm{AF}$ procedures which always has a CS catheter in the field-of-view.

To establish ground truth for evaluation, electrode catheters and guidewires were manually annotated by a clinical expert. An annotated object starts from the edge of the image and ends at its tip. The detection or tracking precision is defined as the average of the shortest distances from points on a detected or tracked object to the corresponding annotated object.

\section{A. Evaluation of catheter and wire detection}

For the technique to be acceptable in clinical practice, failed detections are considered to be the ones where any points on the detected object have larger errors than a preset threshold (e.g., a threshold of $2.5 \mathrm{~mm}$ (around 6 image pixels) [31] is used in this evaluation), which corresponds to the size of the smallest target structures for cardiac catheterization procedures. Overall, detection errors for catheter and guidewires are $0.56 \pm 0.28 \mathrm{~mm}$ and $0.68 \pm 0.33 \mathrm{~mm}$, respectively, and success rates of $91.4 \%$ and $86.3 \%$ were achieved. The success rate is defined as: success rate $=1.0-$ failure rate. The detection errors were calculated using all $(7,754)$ validation images. The number of 
points for error measurements range from 11 to 46 depending on the length of the detected object and the points are the position of tensors. The detection errors are presented in table 1 .

\begin{tabular}{|l|l|l|l|l|l|}
\hline Method & Case & Type & Speed & $\begin{array}{l}\text { Errors } \\
\text { (mm) }\end{array}$ & $\begin{array}{l}\text { Errors } \\
\text { (pixels) }\end{array}$ \\
\hline Our method & EP & Catheter & 11 FPS & $0.41 \pm 0.22$ & $1.1 \pm 0.6$ \\
\hline Our method & CRT & $\begin{array}{l}\text { Catheter } \\
\text { wire }\end{array}$ & 11 FPS & $0.64 \pm 0.24$ & $1.6 \pm 0.6$ \\
\hline Our method & CHD & $\begin{array}{l}\text { Catheter } \\
\text { +wire }\end{array}$ & 11 FPS & $0.76 \pm 0.56$ & $1.8 \pm 1.3$ \\
\hline Chen [12] & CRT & Wire & N/A & $0.47 \pm 0.31$ & $2.2 \pm 1.5$ \\
\hline Yatziv [13] & EP & Catheter & 10 FPS & N/A & $3.0 \pm 4.2$ \\
\hline Wu [16] & EP & Catheter & 5 FPS & 0.5 to 0.76 & N/A \\
\hline $\begin{array}{l}\text { Ambrosini } \\
{[15]}\end{array}$ & Liver & $\begin{array}{l}\text { Catheter } \\
+ \text { wire }\end{array}$ & 8 FPS & 0.2 to 0.9 & N/A \\
\hline
\end{tabular}

Table 1. Detection errors among different procedures as well as comparison to other state-of-the-art methods.

The detection method is robust even when angiography was present nearby. The reason for robust wire reconstruction is mainly because the angiography was filtered out by the vessel enhancement filter. Figure 7 give an example. The maximum errors in CRT and CHD cases are much higher than in the EP cases. This is because the catheter or guidewire tracking is distracted by a nearby wire object, and some catheters used in CRT and CHD do not have electrodes, which make them less distinguishable from other wire objects. Figure 8 gives examples of failure cases for detections. Both figure 7 and 8 has one guiding catheter and one guidewire. The guiding catheter is used for injecting contrast agent.

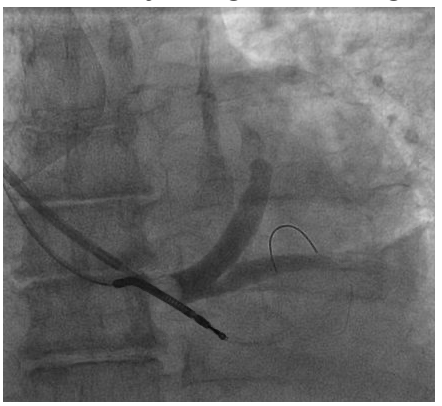

(a)

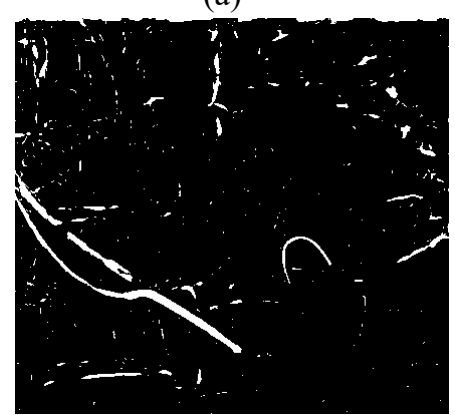

(c)

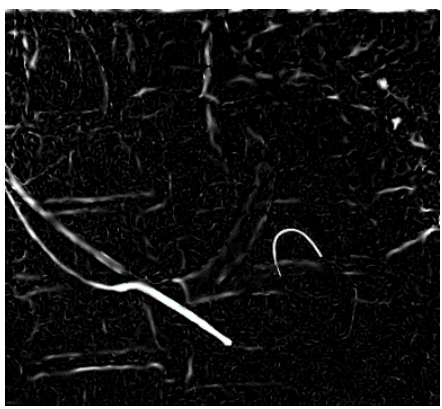

(b)

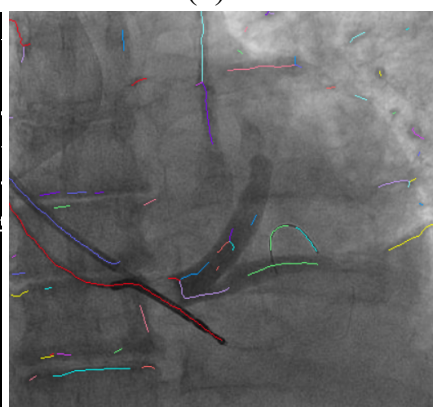

(d)

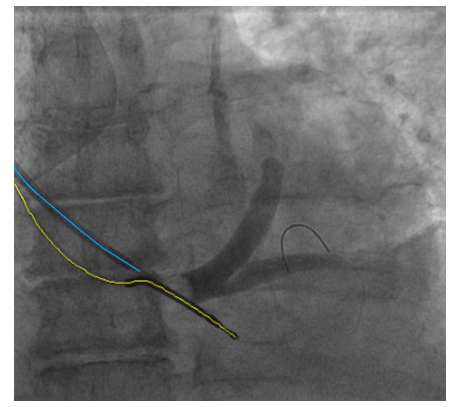

(e)

Fig. 7. The detection result in a CRT case with angiography present. (a) Original image. (b) Image after applying vessel filter. (c) Image after applying binarization. (d) All detected fibres showing in color lines. Based on anatomical information, the two fibres at the top (light blue and purple) are discarded. (e)The yellow line and blue line are the detected wire objects.

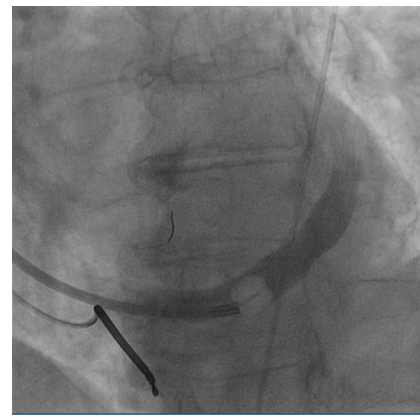

(a)

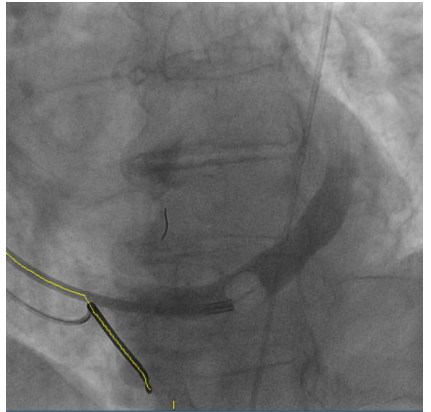

(c)

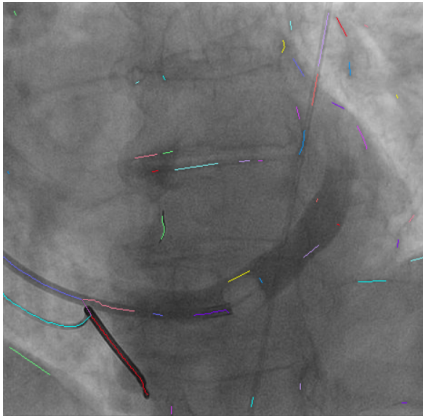

(b)

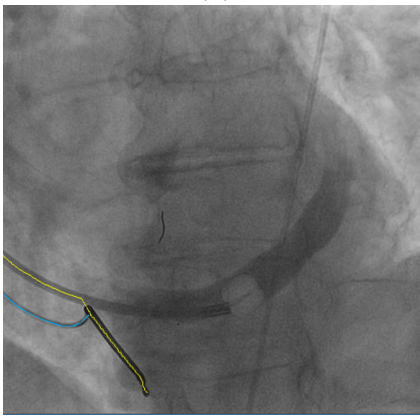

(d)
Fig. 8. A detection failure in a CRT case. (a) Original image. (b) All detected fibres showing in color lines. (c) The yellow line is the detected wire. The failure is caused by a nearby wire object. (d) The yellow line and blue line are detected wire objects.

Overall, tracking errors for catheters and guidewires are 0.41 $\pm 0.23 \mathrm{~mm}$ and $0.58 \pm 0.29 \mathrm{~mm}$, respectively, and success rates of $98.3 \%$ and $90.2 \%$ were achieved. The definition of tracking errors is the same as the definition of detection errors. The success tracking rate is defined as ratio between the number of success tracking cases and total number of tracking cases. As we are tracking using a tracking template, tracking is more robust compared with detection. The rate for obtaining a correct (valid) template is very high as the target catheter or guidewire is stationary and only moves with respiratory and cardiac motions. The success rate for tracking the template is $100 \%, 100 \%$ and $95 \%$ in EP, CRT and CHD cases respectively. A success tracking template is defined as all its points have an error less than $2.5 \mathrm{~mm}$ compared with the manual annotation. The success tracking rate is defined as the percentage of successful tracking templates. Currently, the proposed 
framework has achieved a frame rate of up to 11 frames per second (FPS) in detection and 16 FPS in tracking. The performance was evaluated on an Intel Core i7 $2.9 \mathrm{GHz}$ laptop with a single-threaded CPU implementation.

The tracking errors for both proximal electrode and catheter tip electrode of a CS catheter was given in table 2 .

\begin{tabular}{|l|l|l|l|}
\hline Electrode & Case & Errors $(\mathrm{mm})$ & Errors (pixels) \\
\hline Catheter tip & EP & $0.52 \pm 0.27$ & $1.4 \pm 0.7$ \\
\hline Proximal & EP & $0.43 \pm 0.22$ & $1.2 \pm 0.6$ \\
\hline
\end{tabular}

Table 2. Tracking errors of proximal electrode and catheter tip electrode for a CS catheter.

\section{B. Validation of motion compensation}

A lasso catheter is often used in EP procedures. The lasso catheter is normally placed inside the pulmonary veins (PV) to be used as a mapping or measurement catheter. 18 out of 56 image sequences recorded in the EP cases had a lasso catheter which remained stable in one of the PVs throughout the whole image sequence. Therefore, it can be used for validation of accuracy of motion compensation using CS catheter tracking. The 2D translational motion of the proximal electrode of the CS catheter was applied to the $2 \mathrm{D}$ position of the lasso catheter tip electrode. This acts as a surrogate for the position of the PVs, since it is rigidly placed within these structures during the procedure. The target registration error (TRE) was computed as the distance error between this predicted position of the lasso catheter tip electrode and the actual position of the lasso catheter tip electrode in the X-ray image. Figure 9 gives an example. The positions of the lasso catheter tip were manually annotated by a clinical expert. TRE before motion correction was $4.5 \pm 1.6 \mathrm{~mm}$ and after applying motion compensation using a CS catheter TRE dropped to $1.8 \pm 0.9 \mathrm{~mm}$. The TRE was calculated at the catheter tip electrode on 721 images (14 image sequences and 10 patients). Table 3 summarizes the accuracy and motion recovery of motion compensation validation using a lasso catheter.

\begin{tabular}{|l|l|l|l|}
\hline & Case & Errors (mm) & Errors (pixels) \\
\hline Before correction & EP & $4.5 \pm 1.6$ & $12.1 \pm 4.3$ \\
\hline After correction & EP & $1.8 \pm 0.9$ & $4.8 \pm 2.4$ \\
\hline Motion recovered & \multicolumn{3}{|c|}{$41 \% \sim 71 \%$} \\
\hline
\end{tabular}

Table 3. TRE before and after motion correction using lasso catheter validation.

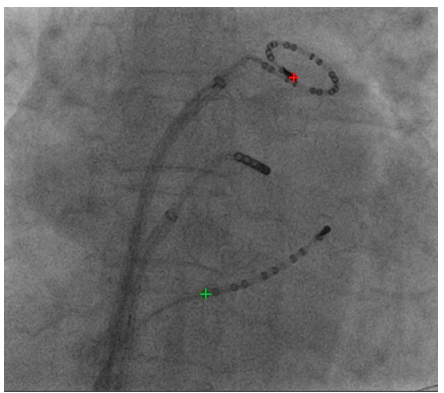

(a)

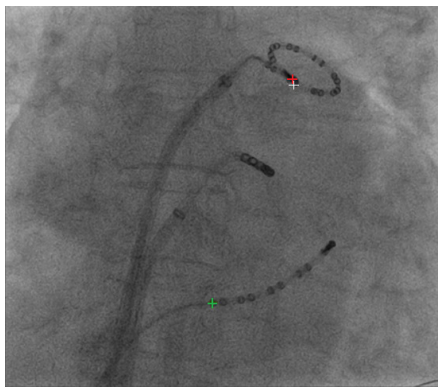

(b)
Fig. 9. Example of motion compensation validation using the lasso catheter. (a)(b) The green cross is the tracked proximal electrode of CS catheter. The red cross is the manually annotated position of lasso catheter tip electrode. (b) The white cross is the predict position of lasso catheter tip electrode.

\section{Validation of $2 D-3 D$ registration}

For validating 2D-3D registration using a CS catheter, two 2D B-spline curves were used to annotate the border of the left atrium roof (LAR) (shown in figure 10f) in the X-ray angiography and the 3D LA model. The TRE was defined as the average $2 \mathrm{D}$ distance between the two B-spline curves. TRE for 2D-3D registration are $2.3 \pm 0.9 \mathrm{~mm}$. Validation was on 5 image sequences (49 image frames from $3 \mathrm{EP}$ cases were used) which had left atrium angiography. The ICP residual error is $0.53 \pm 0.21 \mathrm{~mm}$. Not all image frames in each sequence are used as angiography is only clearly visible on some of image frames. Table 4 summarizes the accuracy of 2D-3D registration validation using LAR.

\begin{tabular}{|l|l|l|l|l|}
\hline Images & Case & $\begin{array}{l}\text { ICP Errors } \\
(\mathrm{mm})\end{array}$ & Errors $(\mathrm{mm})$ & Errors (pixels) \\
\hline 49 & EP & $0.53 \pm 0.21$ & $2.3 \pm 0.9$ & $6.2 \pm 2.4$ \\
\hline
\end{tabular}

Table 4. The ICP residual errors and TRE for LAR validation.

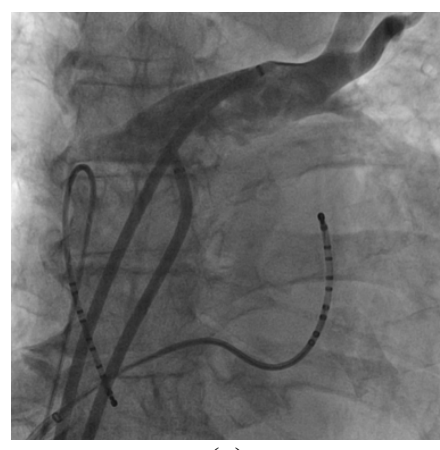

(a)

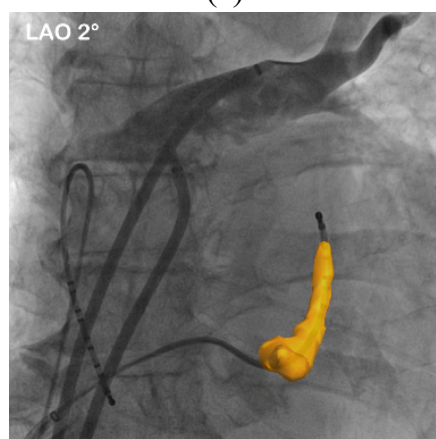

(c)

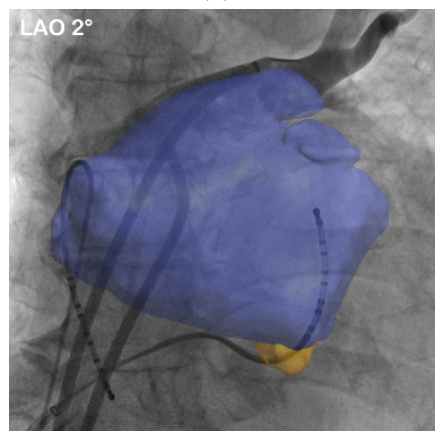

(e)

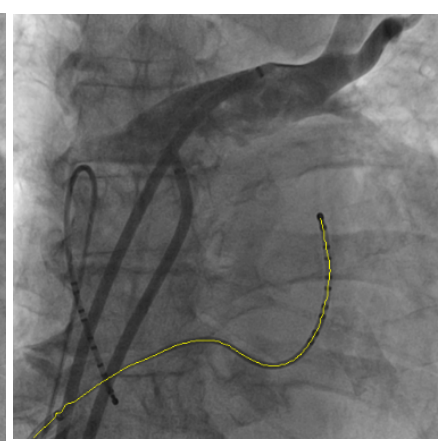

(b)

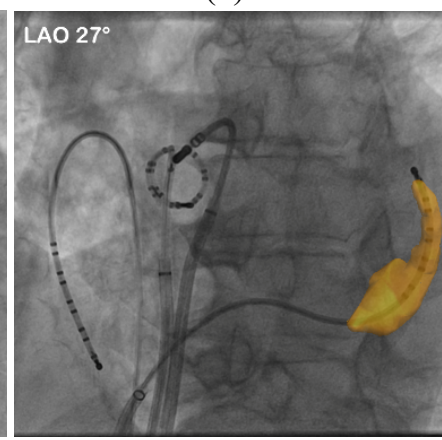

(d)

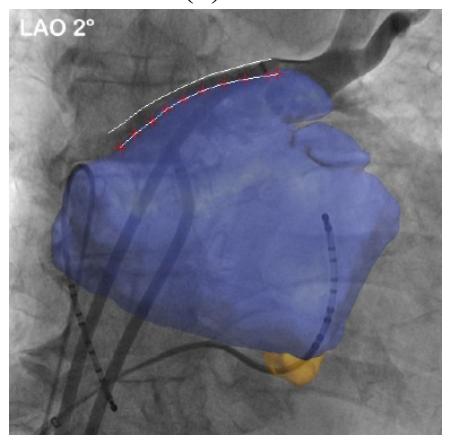

(f)
Fig. 10. Example of an automatic 2D-3D registration and validation. The selection of the target CS catheter (two CS catheters in the image) is automatically based on anatomical information. (a) Original image. (b) CS catheter tracking result. (c) 2D-3D registration result (LAO 2 acquisition angle). Orange object is the 3D model of CS created from the segmentation of the whole heart MRI images. (d) 2D-3D registration result (LAO 27). (e) Left 
Atrium (blue color) and CS (orange color) overlay. (f) Manual annotation (10point B-spline curve) of the LAR used in validating 2D-3D registration methods.

\section{DisCUSSION AND CONCLUSION}

This paper presents a robust real-time catheter and guidewire tracking framework and its novel clinical applications. It utilizes image tensors to reconstruct the path of a catheter or a guidewire which provide a robust tracking template. With automatic pattern detection on an electrode catheter, the target object was automatically detected without any user interaction even when there were multiple catheters presented in the image. With a tracking speed of 16 FPS, the framework can be used for real-time motion compensation, as the average maximum frame rate for modern intervention X-ray systems is 15 FPS. Using the $2 \mathrm{D}$ position of the proximal electrode of a CS catheter can recover up to $71 \%$ of respiratory motion, which is a similar result compared with [32]. This was compared against the static roadmap and the percentage of motion recovered is calculated as

$$
M_{\text {rec }}=100 \% *\left(T R E_{\text {before }}-T R E_{\text {after }}\right) / T R E_{\text {before }}
$$

Our solution is not limited to the CS catheter and it could use any wire inserted into heart vessels or chambers as long as it remains stationary and only moves with respiratory and cardiac motions. The proposed framework can be also used for automatic 2D-3D registration between $3 \mathrm{D}$ anatomical models and live X-ray images. The only requirement for this clinical application is the 3D model of the CS, which could be obtained from a 3D whole-heart MRI scan. The current MRI acquisition protocol of whole-heart images requires the patient to hold their breath for 10 to 20 seconds. Most patients can hold their breath for the required time period but majority of the CRT patients cannot. Therefore, with motion artifacts presented in image data from CRT patients, an accurate CS segmentation is sometimes not possible. The $2 \mathrm{D}-3 \mathrm{D}$ registration method was therefore only

\section{REFERENCES}

[1] I Vernikouskaya et al., "Patient-specific registration of 3D CT angiography (CTA) with X-ray fluoroscopy for image fusion during transcatheter aortic valve implantation (TAVI) increases performance of the procedure", Clinical Research in Cardiology. Vol. 107 no.6, pp507$516,2018$.

[2] J. Ector et al., "Biplane three-dimensional augmented fluoroscopy as single navigation tool for ablation of atrial fibrillation: Accuracy and clinical value", Heart Rhythm, vol. 5, no. 7, pp957-964, 2008.

[3] S. Knecht et al., "Computed tomography-fluoroscopy overlay evaluation during catheter ablation of left atrial arrhythmia", Europace, vol. 10, no, 8, pp931-938, 2008.

[4] Choi J et al., "X-ray and magnetic resonance imaging fusion for cardiac resynchronization therapy", Medical Image Analysis, vol 31, pp98-107, 2016.

[5] T. Geva and A. C. Marshall, "Magnetic Resonance Imaging-Guided Catheter Interventions in Congenital Heart Disease", Circulation, vol. 113 , no. 8, pp 1051-1052, 2006.

[6] M.V. Orlov et al., "Three-dimensional rotational angiography of the left atrium and esophagus: a virtual computed tomography scan in the electrophysiology lab", Heart Rhythm, vol. 4, no. 1, pp37-43, 2007.

[7] A. Al-Ahmad et al., "Time-resolved three-dimensional imaging of the left atrium and pulmonary veins in the interventional suite: A comparison between multisweep gated rotational three-dimensional reconstructed fluoroscopy and multislice computed tomography", Heart Rhythm, vol. 5, no. 4, pp513-519, 2008 tested on image data from EP cases. Automatic registration and motion compensation methods could be applied together throughout the procedure to correct bulk patient motions as well as respiratory motions.

Existing state-of-the-art methods track either only electrodes or only the partial or full length of a catheter or guidewire. Some existing methods requires manual initialization or a prior model. To the best of our knowledge, none of existing methods fulfills all our requirements for our novel clinical applications. On the other hand, our framework can track both catheter and guidewire in real-time and it also detect electrode positions on the catheter. Finally, the full length of a catheter or guidewire is tracked. All features of our framework enable it to be used in motion compensation as well as automatic co-registration between $2 \mathrm{D}$ X-ray images and the $3 \mathrm{D}$ roadmap.

With the rise of robot-assisted intervention in the near future, automatic tracking of surgical and interventional tools has become an important part of the robotic system. For example, in robot-assisted cardiovascular surgery, real-time localization of the tip of the catheters or guidewires in the X-ray image could facilitate force-feedback in robotic controls to avoid damaging blood vessel walls. If the proposed framework integrates more image or object classifiers, there will be wider applications in computer or robot assisted interventions.

\section{ACKNOWLEDGEMENT}

This work was supported by core funding from the Wellcome/EPSRC Centre for Medical Engineering [WT203148/Z/16/Z] and the National Institute for Health Research (NIHR) Biomedical Research Centre at Guy's and St Thomas' NHS Foundation Trust and King's College London. The views expressed are those of the author(s) and not necessarily those of the NHS, the NIHR or the Department of Health.

[8] A.P. King et al., "A subject-specific technique for respiratory motion correction in image-guided cardiac catheterisation procedures", Medical Image Analysis, vol. 13, no. 3, pp419-431, 2009

[9] M. Alhrishy et al., "A machine learning framework for context specific collimation and workflow phase detection", CMBBE, 2018.

[10] A. Brost et al., "3-D respiratory motion compensation during EP procedures by image-based 3-D lasso catheter model generation and tracking”, In: Yang, G.-Z., et al. (eds.) MICCAI 2009, Part I. LNCS, 5761, pp394-401, 2009.

[11] A. Brost et al., "Respiratory motion compensation by model-based catheter tracking during ep procedures", Medical Image Analysis, vol. 14, no. 5, pp695-706, 2010.

[12] B. J Chen et al., "Guidewire tracking using a novel sequential segment optimization method in interventional x-ray videos", Proc IEEE Int Symp Biomed Imaging 2016: 103-106.

[13] L. Yatziv et al., "Toward Multiple Catheters Detection in Fluoroscopic Image Guided Interventions", IEEE Transactions on Information Technology in Biomedicine, vol. 16, no. 4, pp770-781, 2012.

[14] Y. J. Zhou et al., "A Real-time Multi-functional Framework for Guidewire Morphological and Positional Analysis in Interventional X-ray Fluoroscopy," in IEEE Transactions on Cognitive and Developmental System, 2020

[15] P. Ambrosini et al., "Fully Automatic and Real-Time Catheter Segmentation in X-Ray Fluoroscopy" MICCA 2017, LNCS 10434 pp577$585,2017$.

[16] W. Wu et al., "Learning-based Hypothesis Fusion for Robust Catheter Tracking in 2D X-ray Fluoroscopy”, CVPR, 2011:1097 - 1104. 
[17] Y. Ma et al., "Real-time x-ray fluoroscopy-based catheter detection and tracking for cardiac electrophysiology interventions", Medical Physics, vol. 40, no. 7, pp071902-1- 071902-13, 2013.

[18] Y. Ma et al., "A novel real-time computational framework for detecting catheters and rigid guidewires in cardiac catheterization procedures", Medical Physics, vol. 45, no. 11, pp5066-5079, 2018.

[19] O. Pauly et al., "A machine learning approach for deformable guide-wire tracking in fluoroscopic sequences", MICCAI, 2010.

[20] M. Panayiotou et al., "A statistical method for retrospective cardiac and respiratory motion gating of interventional cardiac x-ray images", Medical Physics, vol. 41, no. 7, 2014.

[21] O. Ecabert et al., "Automatic model-based segmentation of the heart in CT images", IEEE Transactions on Medical Imaging, vol. 27, no. 9, pp1189-201, 2008.

[22] J. Peters et al., "Automatic whole heart segmentation in static magnetic resonance image volumes", MICCAI, LNCS 4792, pp402-410, 2007.

[23] A. F. Frangi et al., "Multiscale vessel enhancement filtering", MICCAI, 1998.

[24] N. Otsu, "A threshold selection method from gray-level histograms", IEEE Transactions on Systems, Man and Cybernetics, 9(1), 62-66, 1979.

[25] H. R. Fazlali et al, "Vessel region detection in coronary X-ray angiograms", International Conference on Image Processing, 2015.

[26] I. Dryden et al., "Non-Euclidean statistics for covariance matrices, with applications to diffusion tensor imaging", Ann. Appl. Stat., vol. 3, no. 3, pp1102-1123, 2009

[27] D. Zhou et al., "Regularisation, interpolation and visualisation of diffusion tensor images using non-Euclidean statistics", Journal of Applied Statistics, vol. 43, no.5, pp943-978, 2016.

[28] D.J. Hawkes et al., "The accurate 3-D reconstruction of the geometric configuration of vascular trees from X-ray recordings", Physics and Engineering of Medical Imaging, vol. 119, pp250-256, 1987.

[29] Lee et al. "Building skeleton models via 3-D medial surface/axis thinning algorithms, Computer Vision, Graphics, and Image Processing, vol. 56, no.6, pp462-478, 1994.

[30] Y. L. Ma et al., "Image-Based Automatic Ablation Point Tagging System with Motion Correction for Cardiac Ablation Procedures", IPCAI, pp145$155,2011$.

[31] A. Barbu et al., "Hierarchical learning of curves application to guidewire localization in fluoroscopy", CVPR, 2007.

[32] P Fischer et al., "An MR-Based Model for Cardio-Respiratory Motion Compensation of Overlays in X-Ray Fluoroscopy", IEEE Transactions on Medical Imaging, vol. 37, no. 1, pp 47-60, 2018. 